\title{
Potencialidades do pensamento computacional em sala de aula: um relato de experiência do IF Baiano, campus Catu
}

\author{
Diêgo Pereira da Conceição, Gilvan Martins Durães \\ Instituto Federal de Educação, Ciência e Tecnologia Baiano (IF BAIANO) campus Catu \\ 48110-000 - Catu - BA - Brasil \\ \{diego.conceicao, gilvan.duraes\}@ifbaiano.edu.br
}

\begin{abstract}
Computational Thinking (CT) has attracted the interest of many researchers around the world. In Brazil, there is still a gap in the popularization of CT in basic education. This paper presents a strategy for the dissemination of CT in the academic environment by activities that encourage the understanding and using the pillars of CT: decomposition, pattern recognition, abstraction and algorithms. The proposed activities took place during event F5 Imersão held at IF Baiano, campus Catu, with a Workshop format with duration 4 (four) hours and had six participants, including teachers and students. In the end of the Workshop, it was possible to state that initiatives like this contribute to the diffusion of computational thinking in our country.
\end{abstract}

\section{Resumo}

O Pensamento Computacional (PC) tem atraído o interesse de muitos pesquisadores ao redor do mundo. No Brasil, ainda há uma lacuna na popularização do PC na educação básica. Este artigo apresenta uma estratégia de disseminação do PC no ambiente acadêmico por meio de atividades que estimulam a compreensão e utilização dos pilares do PC: decomposição, reconhecimento de padrões, abstração e algoritmos. As atividades propostas foram aplicadas durante o evento F5 Imersão realizado no IF Baiano, campus Catu, em formato de Workshop com duração de 4 (quatro) horas e teve seis participantes, entre professores e estudantes. Ao final do Workshop foi possível afirmar que iniciativas como esta contribuem com a difusão do pensamento computacional em nosso país.

\section{Introdução}

O Pensamento Computacional (PC) pode ser entendido como um conjunto de habilidades utilizadas para resolver problemas complexos de diversas áreas do conhecimento, não apenas da ciência da computação. 
No universo da resolução de problemas, o PC é organizado em quatro pilares (BBC, 2018; BRACKMANN, 2017): Decomposição, Reconhecimento de Padrões, Abstração e Algoritmos. A definição de cada um dos pilares do PC é apresentada em seguida.

Decomposição: ao lidar com um problema quebre-o em partes menores para minimizar a complexidade. Reconhecimento de padrões: possivelmente o problema em questão possui semelhanças com outros problemas já resolvidos. Encontrar padrões de resolução já consolidados é essencial para evitar a reinvenção e consequentemente evitar gasto com tempo e outros recursos. Abstração: focar apenas nos aspectos mais importantes do problema sem se preocupar com todos os detalhes. Algoritmos: consolidação dos passos utilizados na resolução do problema. É a descrição de todos os caminhos percorridos desde a abordagem até a solução do problema. Em níveis mais avançados, recomenda-se a utilização do computador e uma linguagem de programação para potencializar as soluções por meio da construção de programas de computador.

Esses pilares podem ser trabalhados de várias maneiras como atividades sem o uso das tecnologias (atividades “desplugadas”), programação Scratch, criação de jogos, robótica pedagógica, produção de narrativas digitais e uso de simulações, como apresentado em Valente (2016).

Os pesquisadores estadunidenses Yadav, Stephenson e Hong (2017) afirmam que, para se ensinar o PC, inicialmente é necessário que haja uma parceria entre professores da ciência da computação com os professores provenientes de outras áreas. Além disso, os autores destacam que os cursos ofertados não devem se limitar ao uso do computador, mas devem ser executados de forma relevante para que haja o desenvolvimento de habilidades e competências desses profissionais nas suas experiências com o PC. No Brasil, pesquisas têm sido realizadas no âmbito da inserção do Pensamento Computacional e da Computação no currículo da Educação Básica (SBC, 2017; BRACKMANN, 2017; VALENTE, 2016).

O trabalho de Silva, Silva e França (2017) traz uma experiência realizada na disciplina Estágio Curricular V do curso de Licenciatura em Computação da UFRPE. Foi realizado um curso com $16 \mathrm{~h}$ de duração com 13 (treze) professores do Centro de Tecnologia na Educação (CETEC) da cidade de Recife com o objetivo de apresentar o conceito do PC por meio de atividades lúdicas. Foram utilizadas atividades que envolviam a computação "desplugada", o jogo LightBot e a plataforma Scratch, estas duas últimas voltadas para o ensino de lógica de programação. O curso foi dividido em quatro encontros, tendo cada um deles $4 \mathrm{~h}$ de duração, objetivos a serem alcançados e os recursos que seriam utilizados. Ao final do curso, os professores responderam a um questionário de 14 (catorze) perguntas, nas quais, foi possível verificar que $100 \%$ dos participantes compreenderam melhor o termo PCe consideraram que a formação foi útil no seu desenvolvimento pessoal. Além disso, $70 \%$ deles apontaram o Scratch como a ferramenta mais viável para utilização nas suas práticas pedagógicas. 
No estudo exploratório de Cavalcante et al. (2017) é investigado como se dá a percepção do pensamento computacional pelos professores do Ensino Médio e se as atividades desenvolvidas por eles em sala de aula estimulam o desenvolvimento dessa habilidade. Eles realizaram um survey com 11 (onze) questões, do qual participaram 15 (quinze) professores, com a finalidade de identificar quais aspectos do pensamento computacional já eram trabalhados pelos professores nas aulas. Em seguida realizaram uma entrevista com 6 (seis) professores selecionados dentre os 15, a fim de compreenderem mais detalhadamente como se dão a realização de tais atividades. Eles concluem que as habilidades do pensamento computacional ainda são poucas exploradas por professores do Ensino Médio devido à falta de conhecimento ou ideia equivocada sobre a temática. Argumentam ainda que a falta de uma formação adequada em pensamento computacional para professores da educação básica seja um impasse para sua utilização nas atividades pedagógicas.

Raabe et al. (2017) apresentam os primeiros resultados da implantação do PC como disciplina obrigatória no Ensino Fundamental e Médio no Colégio de Aplicação da Universidade do Vale de Itajaí (UNIVALI). O público-alvo foi formado por estudantes do $6^{\circ}$ ano do Ensino Fundamental até o $3^{\circ}$ do Ensino Médio, distribuídos em 25 turmas, num total de aproximadamente 700 estudantes. As aulas foram ministradas por 4 (quatro) professores bacharéis em Ciência da Computação que relataram os benefícios do componente curricular no tocante à motivação dos alunos e ao auxílio na compreensão de conteúdos de outras disciplinas como Matemática e Física.

Este trabalho tem como objetivo investigar a percepção de professores e estudantes das diversas áreas do conhecimento sobre o ensino do PC através de atividades plugadas e desplugadas, lançando ideias que tornem possível o seu uso no cotidiano escolar. Desta forma, é apresentado neste trabalho o relato de experiência da realização de um Workshop com duração de 4 (quatro) horas no IF Baiano campus Catu, intitulado "Potencialidades do Pensamento Computacional em sala de aula". As inscrições foram online, gratuitas e abertas ao público interno e externo, sendo direcionado a professores e estudantes de qualquer área do conhecimento.

A seguir, na Seção 2 é descrito o procedimento metodológico adotado durante a realização do Workshop. Na Seção 3 são apresentados os resultados coletados por meio de formulário online e a discussão feita pelos pesquisadores. Por fim, a Seção 4 apresenta a conclusão e as considerações sobre o trabalho desenvolvido.

\section{Procedimentos metodológicos}

A metodologia utilizada no trabalho é dividida em 3 etapas: levantamento bibliográfico, planejamento e realização das atividades e análise avaliativa das atividades. Na etapa primeira etapa foi realizado o levantamento bibliográfico de publicações sobre PC no Portal 
de Periódicos da CAPES ${ }^{1}$ e em sites internacionais ${ }^{2}$ a fim de conhecer como o PC vem sendo trabalhado e discutido ao longo dos anos, dando ênfase às publicações que utilizaram oficinas como meio de ensino. Observou-se nesta etapa inicial que a maioria dos trabalhos publicados na área focam apenas no pilar Algoritmos ou na junção de todos os pilares para a construção de algoritmos. Na segunda etapa, definiu-se então que cada pilar do PC seria trabalhado com uma atividade distinta. Dessa forma, durante o planejamento ficou acordado entre os professores proponentes que para as atividades sobre Decomposição e Algoritmos seriam utilizados materiais e recursos já existentes e disponibilizados gratuitamente na internet, enquanto as atividades de Reconhecimento de Padrões e Abstração seriam elaboradas pelos autores. Por fim, a análise avaliativa se deu por meio de formulário online com perguntas sobre a percepção dos participantes em relação às atividades desenvolvidas durante o Workshop.

O Workshop intitulado "Potencialidades do pensamento computacional em sala de aula" aconteceu no IF Baiano campus Catu durante o evento F5 Imersão e teve uma duração de 4h. O Workshop foi ministrado por dois professores de Informática e teve a participação de 6 (seis) pessoas: uma professora EBTT de Matemática, uma professora da Educação Infantil, um intérprete de Libras, uma estudante do curso de Cozinha na modalidade PROEJA, uma estudante de graduação em engenharia e um ex-aluno do Ensino Médio Integrado. Vale ressaltar que as inscrições para o curso se deram de forma online e limitada a um público de 40 participantes.

$\mathrm{Na}$ Introdução do Workshop foram utilizados slides projetados via datashow para explicar o conceito de Pensamento Computacional, as possibilidades de aplicação no ensino e uma visão panorâmica da educação no Brasil. Em seguida, foram realizadas quatro atividades para contemplar os quatro pilares do PC: Decomposição, Reconhecimento de Padrões, Abstração e Algoritmos. O objetivo foi ilustrar como cada um dos pilares pode ser trabalhado de forma individual em determinadas tarefas. Apesar da relação entre os quatro aspectos do PC, não existe uma ordem específica ou a obrigatoriedade de que eles sejam abordados em conjunto. Assim, o curso foi planejado visando deixar claro aos participantes o que é cada aspecto do PC, possibilitando a ampliação de sua aplicação no planejamento e resolução das atividades acadêmicas.

\subsection{Decomposição}

Para a atividade de decomposição foi entregue aos participantes um exercício sugerido por Krauss e Prottsman (2016). O objetivo principal é criar equações matemáticas baseadas nas imagens, como ilustrado na Figura 1. O problema consiste em determinar quantas faces X,

\footnotetext{
${ }^{1}$ https://www.periodicos.capes.gov.br/

${ }^{2}$ Alguns dos sites acessados para consulta incluem iniciativas da Google e da Code.org $<$ https://edu.google.com/resources/programs/exploring-computationalthinking/><https://code.org/curriculum/course3/1/Teacher>
} 
$\mathrm{Y}$ e $\mathrm{Z}$ existe em cada imagem da atividade. O primeiro exercício foi resolvido junto aos participantes para exemplificar como se dariam os passos da resolução do problema.

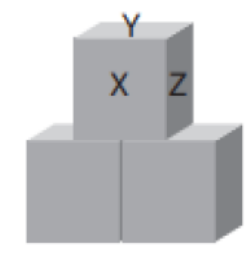

Figura 1. Atividade sobre decomposição.

Fonte: Krauss e Prottsman (2016).

A atividade foi iniciada com um problema menor e relativamente simples com avanços graduais na dificuldade das questões como pode ser visto na Figura 2. As soluções das propostas consistem em perceber que é possível estruturar uma expressão matemática para grupos menores de cubos a exemplo do XYZ e calcular a quantidade de lados separadamente. Seguindo esse raciocínio teríamos as seguintes soluções para o exemplo da figura 1: como temos 2 lados de $X, 2$ lados de $Y$ e 2 lados de $Z$ para um cubo, para 3 cubos idênticos temos $\mathrm{L}=3 * 2 \mathrm{X}+3 * 2 \mathrm{Y}+3 * 2 \mathrm{Z}$, totalizando 6 lados para cada face do cubo. Dessa forma, independentemente de quantos cubos a questão apresenta, a decomposição do problema geral em problemas menores facilita o caminho da solução.

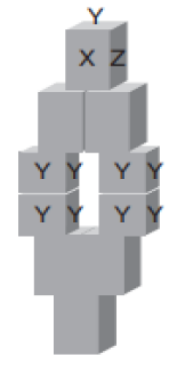

Figura 2. Decomposição - nível mais avançado do problema.

Fonte: Krauss e Prottsman (2016).

\subsection{Abstração}

$\mathrm{Na}$ atividade de abstração foi utilizado o trecho "Ensinar exige comprometimento" do livro "Pedagogia da Autonomia" de Paulo Freire. O texto de 37 linhas distribuídas em 3 parágrafos foi projetado via datashow para uma leitura em conjunto. Em seguida, foi entregue caneta e papel e solicitado aos participantes que respondessem às seguintes questões: Escolha 5 palavras-chave para o texto; Qual o tema principal do texto?; Quais outros assuntos são tratados pelo autor?; A que conclusão o autor chega?; Faça um resumo de até 10 linhas sobre o texto lido.

Essa atividade recorrente no cotidiano de todo estudante consiste em fazer resumos para auxiliar no entendimento de um texto. A atividade de resumos é um bom exemplo do 
uso da abstração, pois permite ao leitor visualizar as partes de um todo dedicando atenção a determinados aspectos do texto.

\subsection{Reconhecimento de padrões}

Para essa etapa do Workshop, foi lançado um desafio aos participantes: eles deveriam decodificar uma mensagem criptografada. A Figura 3 mostra a mensagem criptografada pela cifra de César ${ }^{3}$ e que deveria ser traduzida numa mensagem legível, entendível em nosso alfabeto.

Para ilustrar como seria possível a resolução desse desafio, foi solicitado aos participantes que jogassem uma rodada do "Jogo da forca" antes de iniciarem a atividade. Após o acerto da palavra do jogo, foi questionado aos participantes quais as primeiras letras que lhes vieram à mente e eles responderam a letra "a", seguida das letras "o" e "e", sem ordem de precedência entre estas últimas. A partir dessa observação, foi explicado que eles(as) escolheram essas letras porque implicitamente sabem que elas aparecem na maioria das palavras do nosso alfabeto.

Mensagem codificada:

\section{ydprv dsuhqghu vreuh shqvdphqwr frpsxwdflrqdo $h$ frpr hoh srgh vhu xwlolcdgr qd vdod gh dxod}

Figura 3. Mensagem codificada com a cifra de César.

Fonte: Próprios autores (2020).

A partir disso, foi apresentada uma tabela contendo todas as letras do alfabeto e com que frequência elas aparecem no alfabeto brasileiro. A Figura 4 mostra um recorte da tabela, onde é possível identificar, além das letras e a frequência de cada uma delas, espaços em branco para o preenchimento por parte dos participantes. A resolução da atividade foi orientada da seguinte forma: primeiramente cada letra deveria ser contada e registrada a quantidade de ocorrências na coluna "Quantidade encontrada"; em seguida, esse valor deveria ser dividido pela quantidade total de caracteres da mensagem, multiplicado por 100 e o resultado escrito na coluna "Porcentagem"; e finalmente, o participante compararia a porcentagem de cada letra encontrada na mensagem com a porcentagem da frequência das letras no alfabeto e, por associação, assumir quais as letras com frequências iguais ou próximas deveria ser equivalentes.

Para exemplificar, ao analisar a mensagem codificada, percebe-se que a letra " $d$ " aparece 11 vezes. Como o texto possui um total de 77 caracteres sem espaço, conclui-se que essa letra corresponde a 14,29\% do total de caracteres. Dessa forma, ao consultar a tabela, a

\footnotetext{
${ }^{3}$ A cifra de César é uma técnica primitiva de criptografia e consiste em trocar uma letra do alfabeto por outra que está distante de 3 posições à sua frente.
} 
letra "d" na mensagem codificada é uma forte candidata a representar a letra "a" no alfabeto original.

Essa técnica conhecida como "frequência de letras" torna-se mais precisa quanto mais caracteres tem a mensagem. Devido ao tamanho de apenas 77 caracteres, os professores auxiliaram os participantes na confirmação de algumas letras encontradas que apresentavam a mesma frequência, o que gerava dúvidas. Por fim, o grupo trabalhou junto e conseguiu decifrar a mensagem: "Vamos aprender sobre pensamento computacional e como ele pode ser utilizado na sala de aula”.

\begin{tabular}{|c|c|c|c|c|}
\hline Letra & $\begin{array}{c}\text { Frequência } \\
\text { em nosso } \\
\text { alfabeto }\end{array}$ & $\begin{array}{c}\text { Quantidade } \\
\text { encontrada }\end{array}$ & $\begin{array}{c}\text { Porcentagem } \\
\%\end{array}$ & $\begin{array}{c}\text { Possível } \\
\text { letra }\end{array}$ \\
\hline A & $14,63 \%$ & & & \\
\hline B & $1,04 \%$ & & & \\
\hline C & $3,88 \%$ & & & \\
\hline D & $4,99 \%$ & & & \\
\hline E & $12,57 \%$ & & & \\
\hline
\end{tabular}

Figura 4. Frequência de letras do alfabeto brasileiro.

Fonte: Próprios autores (2020).

\subsection{Algoritmos}

O último momento do Workshop foi realizado no laboratório de informática, onde foi trabalhado o conceito de Algoritmos. Foi utilizada a construção do jogo Flappy Bird disponibilizado gratuitamente na plataforma Code.org, através da programação em blocos, recomendada para pessoas iniciantes na área da computação.

O objetivo da atividade foi apresentar a estruturação e organização do pensamento em passos definidos para alcançar uma solução. No jogo Flappy Bird, o objetivo é fazer com que o pássaro passe por uma série de obstáculos utilizando apenas o click do mouse. A programação do jogo consiste em 10 etapas, na qual cada uma dessas etapas possui instruções do que deve ser feito para que o jogador avance na programação.

Na Figura 5 é possível visualizar que a instrução da etapa 1 é "Conecte um bloco no bloco 'quando clicar' e, em seguida, clique em 'Executar'. Clique ou toque na tela para mover o passarinho em direção ao alvo”. Na área de blocos tem-se os blocos necessários para serem posicionados corretamente na Área de trabalho.

A atividade foi projetada no quadro branco via datashow, com a seguinte dinâmica: enquanto um professor explicava a atividade e realizava o passo a passo do exercício junto à turma, o outro professor auxiliava os participantes individualmente tirando suas dúvidas. 


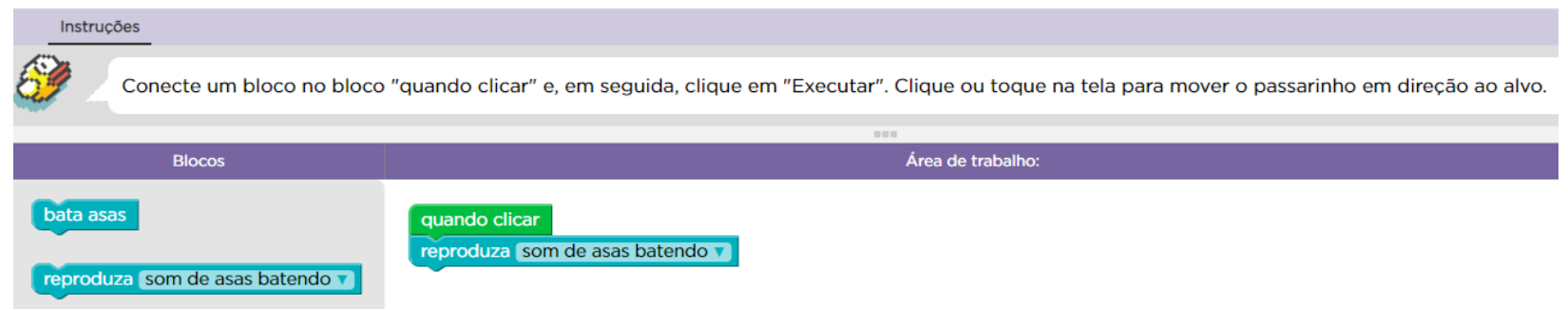

Figura 5. Programação do jogo Flappy Bird.

Fonte: Code.org (2020).

\section{Resultados e Discussão}

Ao final do Workshop foi solicitado aos participantes que respondessem a um formulário online ()para que eles pudessem contribuir para a avaliação do Workshop. Dos 6 participantes que iniciaram o curso, 5 responderam ao questionário.

Ao questionar se o participante sentiu falta de algum conhecimento prévio para compreender os conteúdos do Workshop, as respostas apontaram que não houve a necessidade de conhecimento prévio e que "o conteúdo foi bastante interativo e dinâmico". É importante ressaltar que o planejamento das atividades levou em consideração a diversidade dos participantes e que esse aspecto é fundamental ao trabalhar o PC, uma vez que o pesquisador não deve assumir uma postura de transmissor do conhecimento, mas enriquecer a prática pedagógica juntamente com os envolvidos no processo.

Sobre as dificuldades em relação às atividades práticas, apenas um participante informou ter sentido dificuldades no momento em que foi para o computador realizar a atividade de Algoritmos na programação do jogo Flappy Bird. Os demais participantes informaram que não tiveram dificuldades. O resultado dessa pergunta ainda reflete uma dura realidade brasileira quanto ao acesso das pessoas aos recursos tecnológicos como computadores. Por isso, é essencial que as pesquisas sobre PC realizadas no Brasil continuem enveredando por soluções didáticas que não se limitam ao uso do computador, possibilitando assim, a difusão do conhecimento em suas diferentes formas e acesso pelos professores e estudantes nas diversas modalidades de ensino.

Ao perguntar se o participante tinha mais alguma consideração sobre a metodologia adotada para o processo de ensino-aprendizagem e as atividades utilizadas, um participante disse que o tempo foi curto e outro participante disse "Como é uma perspectiva nova, parabenizo pelo olhar investigativo que está sendo conduzido".

A fim de avaliar os recursos e materiais utilizados, foi solicitado aos participantes que realizassem uma classificação de acordo a clareza/objetividade, profundidade adequada e atratividade. Como mostra a Figura 6, todos os participantes consideraram que sempre houve clareza/objetividade e atratividade nos materiais disponibilizados; e quanto à profundidade, 3 consideraram que sempre houve, e 2 na maioria das vezes. As respostas a essa pergunta 
refletem a metodologia utilizada na elaboração e realização das atividades, pois no início de cada uma delas era apresentado o objetivo e como proceder com a resolução, bem como, ao final, qual aspecto do PC estava sendo abordado.

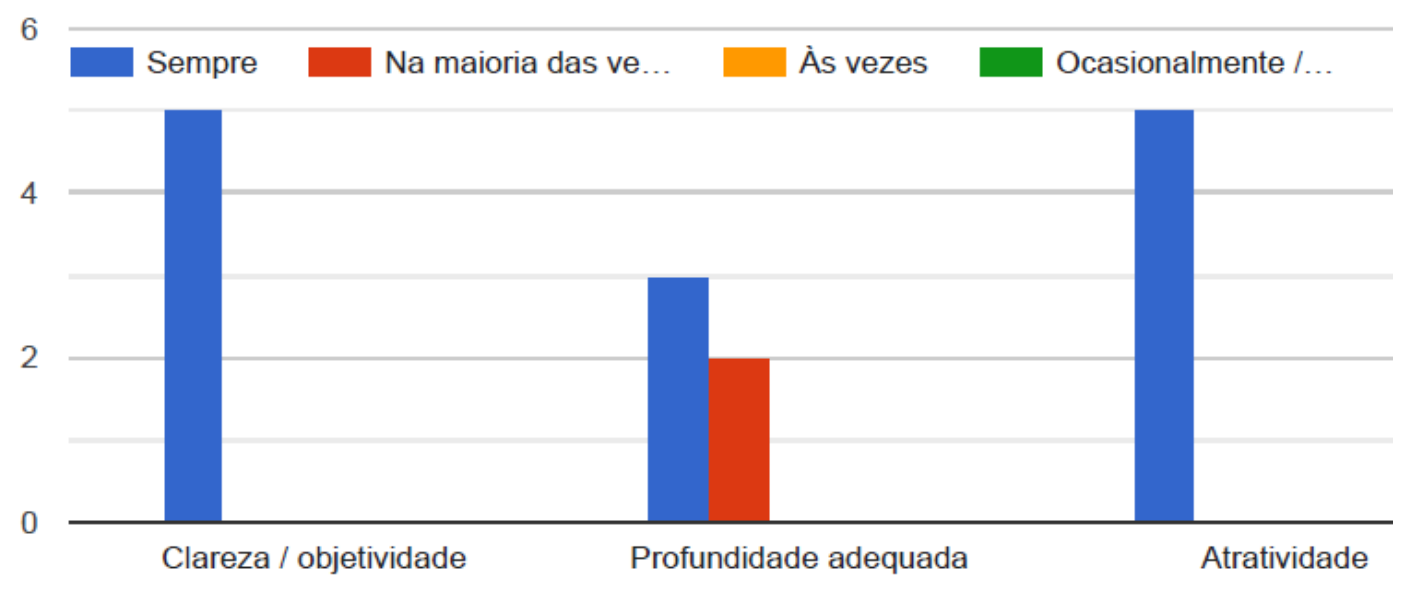

Figura 6. Avaliação dos recursos e materiais utilizados.

Fonte: Próprios Autores (2020).

Como o Workshop foi planejado para diferentes áreas do conhecimento, foi questionado se durante o curso houve o auxílio à compreensão das potenciais aplicações do conteúdo a área de interesse do participante. Todos afirmaram que sim, o tempo todo. Um dos objetivos deste Workshop foi mostrar o uso de atividades que não necessitam obrigatoriamente do computador e que não estejam obrigatoriamente relacionadas ao raciocínio lógico e realização de cálculos, mostrando dessa forma aos professores e estudantes o potencial do PC no cotidiano escolar.

Dentre os quatro pilares do PC trabalhados no Workshop, foi perguntado sobre qual desses conceitos o participante teve mais facilidade em compreender. $\mathrm{O}$ resultado foi diversificado, tendo 2 participantes indicado Abstração e 1 participante para cada conceito restante, decomposição, reconhecimento de padrões e algoritmos. Essa diversificação no resultado é compreensível, pois o público possuía formações e áreas de interesses variadas.

\section{Conclusões}

São inúmeras as possibilidades do desenvolvimento de atividades para trabalhar o PC. Ao realizar o levantamento bibliográfico foi possível constatar que os pesquisadores têm buscado alcançar todas as modalidades de ensino desde a educação infantil, utilizando da pesquisa científica na elaboração de recursos e materiais didáticos para o ensino do PC.

O Workshop aqui apresentado contribui nesse sentido, ao ampliar as perspectivas de aplicações do PC para professores e estudantes. Devido ao desconhecimento sobre a formação dos participantes, as atividades foram desenvolvidas de forma a contemplar as mais diversas modalidades de ensino e área do conhecimento, para professores e estudantes. Ao 
final do Workshop, foi possível perceber que a escolha das atividades se mostrou adequada, promovendo a compreensão do que é o PC e como ele pode ser utilizado no cotidiano escolar. Dessa forma, espera-se que a divulgação dessa experiência contribua com a popularização do Pensamento Computacional em nosso país, de forma a envolver não apenas professores e estudantes da área da Computação.

\section{Referências}

BBC (2018). "Introduction to computational thinking", https://www.bbc.co.uk/bitesize/guides/zp92mp3/revision/1, Novembro.

BRACKMANN, Christian Puhlmann. (2017). Desenvolvimento do pensamento computacional através de atividades desplugadas na educação básica. $\mathrm{PhD}$ thesis, Universidade Federal do Rio Grande do Sul.

CAVAlCANTE, A. F; COSTA, L. S; ARAúJO, A. L. S; ANDRADE, W. L; GUERRERO, D. D. S. (2017). Um Estudo Exploratório da Aplicação de Pensamento Computacional Baseado nas Perspectivas de Professores do Ensino Médio. In Anais dos Workshops do VI Congresso Brasileiro de Informática na Educação (WCBIE).

KRAUSS, J; PROTTSMAN, K. (2016), Computational Thinking and Coding for Every Student: The Teacher's Getting-Started Guide, Corwin.

RAABE, A. L. A et al. (2017). Experiência de Implantação de uma Disciplina Obrigatória de Pensamento Computacional em um Colégio de Educação Básica. In Anais dos Workshops do VI Congresso Brasileiro de Informática na Educação (WCBIE).

SBC (2017). "Referenciais de Formação em Computação: Educação Básica", https://www.sbc.org.br/files/ComputacaoEducacaoBasica-versaofinal-julho2017.pdf, Novembro.

SILVA V; SILVA, K; FRANÇA, R. S. (2017). Pensamento computacional na formação de professores: experiências e desafios encontrados no ensino da computação em escolas públicas. In Anais dos Workshops do VI Congresso Brasileiro de Informática na Educação (WCBIE).

VALENTE, Jorge Armando. (2016). Integração do Pensamento Computacional no

Currículo da Educação Básica: Diferentes Estratégias Usadas e Questões de Formação de Professores e Avaliação do Aluno. In Revista eCurriculum, v.14, n.3, p.5562.

WING, J. M. (2006). Computational Thinking. In Communications of the ACM, v.49, n. 3, p. 33-35.

YADAV, A., STEPHENSON, C., HONG, H. (2017). Computational Thinking for Teacher Education. In Comunications of the ACM, v. 60, n. 4, p.55-62. 